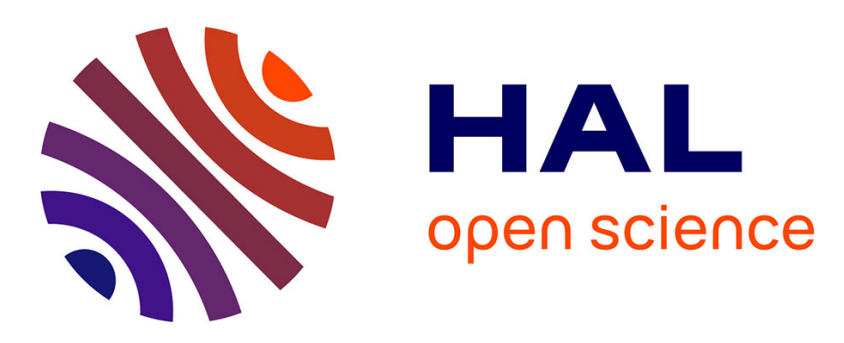

\title{
Graph Coloring Based Pilot Reuse Among Interfering Users in Cell-Free Massive MIMO
}

Wafa Haj Hmida, Jean Pierre Cances, Wafa Haj Hmida, Vahid Meghdadi, Ammar Bouallegue, Jean-Pierre Cances

\section{To cite this version:}

Wafa Haj Hmida, Jean Pierre Cances, Wafa Haj Hmida, Vahid Meghdadi, Ammar Bouallegue, et al.. Graph Coloring Based Pilot Reuse Among Interfering Users in Cell-Free Massive MIMO. ICC 2020, Jun 2020, Dublin, Ireland. pp.1-6, 10.1109/ICCWorkshops49005.2020.9145111 hal-02528981

\section{HAL Id: hal-02528981 \\ https://hal.science/hal-02528981}

Submitted on 2 Apr 2020

HAL is a multi-disciplinary open access archive for the deposit and dissemination of scientific research documents, whether they are published or not. The documents may come from teaching and research institutions in France or abroad, or from public or private research centers.
L'archive ouverte pluridisciplinaire HAL, est destinée au dépôt et à la diffusion de documents scientifiques de niveau recherche, publiés ou non, émanant des établissements d'enseignement et de recherche français ou étrangers, des laboratoires publics ou privés. 


\section{Graph Coloring Based Pilot Reuse Among Interfering Users in Cell-Free Massive MIMO}

\author{
$1^{\text {st }}$ Wafa Haj Hmida \\ University of Tunis El Manar, \\ Tunis, Tunisia \\ wafa.haj_hmida@unilim.fr
}

\author{
$2^{\text {nd }}$ Vahid Meghdadi \\ XLIM, UMR 7252, \\ Limoges, France \\ meghdadi@ensil.unilim.fr
}

\author{
$3^{\text {rd }}$ Ammar Bouallegue \\ University of Tunis El Manar, \\ Tunis, Tunisia \\ ammar.bouallegue@enit.rnu.tn
}

\author{
$4^{\text {th }}$ Jean-Pierre Cances \\ XLIM, UMR 7252, \\ Limoges, France \\ cances@ensil.unilim.fr
}

\begin{abstract}
In cell-free massive multiple-input-multiple-output (CF-mMIMO) systems, we spread a massive number of controllable access points out over a coverage area to simultaneously serve much smaller number of user equipments (UEs) over the same time/frequency resources. The major objective of coining such systems is to offer to all UEs a fair quality of service, avoiding the high interference for cell-edge UEs in cellular networks. However, this is degraded by the pilot contamination problem due to the inter-user interference (IUI). In addition, contrary to the centralized mMIMO, CF-mMIMO is characterized by a channel hardening not sufficiently pronounced, thus it will be appropriate to include downlink (DL) pilots to estimate the DL channel. In this paper, we evolve a graph coloring algorithm for DL pilot assignment in CF-mMIMO system (GC-PA-CFmMIMO) to mitigate the DL pilot contamination. Especially, by exploiting IUI in DL, a conflict or interference graph aims to model the potential interference among interfering users. Then, GC-PA-CF-mMIMO mitigates the potential IUI in DL by appointing adequate pilots among UEs in the conflict graph. The simulation results validate the proposed approach and reveal that, remarkably, it outperforms the DL pilot assignment methods based on prior approachs in terms of min-per-user DL net throughput.
\end{abstract}

\section{INTRODUCTION}

Cell-free massive MIMO (CF-mMIMO) is a particular deployment of distributed mMIMO where the large number APs, equiped with one or multiple antennas, are located within an unlimited area (no cells) [1]. Fronthaul connections are involved to link the APs to central processing units (CPUs), which are connected via backhaul network, if there are multiple CPUs. The benefits to opt for CF-mMIMO are: (i) improving spatial multiplexing by increasing the macrodiversity gain; (ii) reducing the inter-user interference (IUI): when we have a distributed topology, more directive and spatially focused beams result in less IUI especially for celledge UEs who suffer from very high interference [2]; and (iii) offering a very high coverage probability and improving energy efficiency [3]. However, this leads to higher fronthaul requirements and the need for distributed signal processing. Another feature characterising the CF-mMIMO is its low degree of channel hardening owing to its distributed architecture [4], [5]. The UEs, having low degree of channel hardening, rely on beamformed DL pilots to decode its intended data [4].

The use of DL pilots will overwhelm the radio resources at the price of data. Hence, using pilot assignment strategies become relevant to reduce the pilot overhead and to mitigate the DL pilot contamination in CF-mMIMO. Reference [4] considered a pilot utility metric to decide whether a UE needs to receive an orthogonal DL pilot to acquire the DL channel gain or just rely on statistical channel to guarantee higher DL per-user throughput. However, in practice, since the length of the coherence interval is limited, we should reduce the pilot overhead by reusing pilots or using non-orthogonal pilots. In this respect, [1], [6] used random pilot assignment. On the one hand, its strongest part is the simplicity and the susceptibility to be used in distributed networks. On the other hand, its weakest part is that it does not take into consideration the case where UEs in close vicinity use the same pilot. This leads to severe pilot contamination in CF-mMIMO.

Greedy pilot assignment algorithm in [1] allows to assign randomly uplink (UL) pilots to all UEs. Then, this assignment is improved iteratively by selecting the UE corresponding to the minimum per-user DL rate. The selected UE updates its UL pilot so that its pilot contamination effect is mimimized. Moreover, [7] proposed a joint UL-DL pilot assignment algorithm to minimize a utility function taking into consideration the UL and DL pilot contamination ensuring uniform quality of service throughout the network. Another method to cope with reducing pilot overhead is the clustering pilot assignment. A regular pilot reuse scheme is adopted in [8] to guarantee that UEs sharing the copilot are enough geographically separated, and to keep the pilot contamination at an acceptable low level. Moreover, to control the pilot contamination in CF-mMIMO, [9] proposed a dynamic cooperation clustering, which is a user-centric approach where each UE is served by the AP subset providing the best channel conditions. Furthermore, the graph theoretic approach was used to assign DL pilots in order to reduce the DL pilot overhead in a single-cell mMIMO [10]. By exploiting the large-scale fading coefficients, [11] studied the UL interference effect that one UE can impact on another UE when sharing the same UL pilots in multi-cell mMIMO. In such systems, DL pilots are not needed thanks to the channel reciprocity and the channel hardening. This is valid under the assumption of low/moderate speed user. Assuming low degree of channel hardening and high-speed UEs, the study in [11] can be extended to the case of DL interference in CF-mMIMO. Moreover, it will be appropriate to determine under which conditions UEs can reuse the same DL pilot without causing DL pilot contamination. 


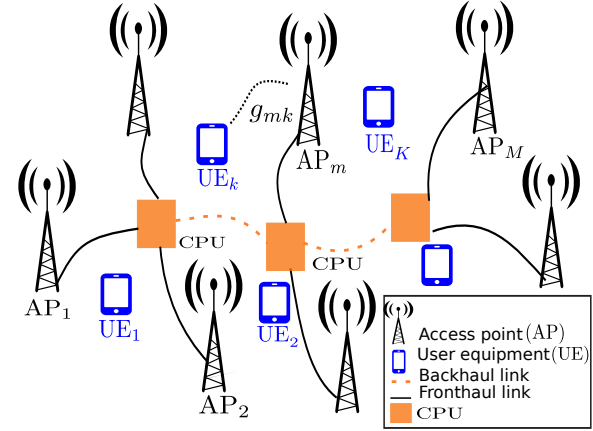

Fig. 1: Cell-free massive MIMO (CF-mMIMO) architecture.

In this paper, the innovation and novelty are orientated towards the use of coloring graph for DL pilot assignment in CF-mMIMO to mitigate the DL pilot contamination. Specifically, inspired by [11], our contributions are laser-focused on proposing a metric to measure the strength of potential interuser interference between two UEs. Then, we construct an interference graph to describe the potential IUI relationship among all UEs in CF-mMIMO. Moreover, we propose a graph coloring algorithm to greedly assign different pilots to the connected UEs. Finally, we compare the performance gain of the proposed scheme to the litterature.

\section{CELL-FREE MASSIVE MIMO SYSTEM MODEL}

The system model adopted in this work is a CF-mMIMO system. It consists of $M$ controllable single-antenna APs and $K$ single-antenna UEs, which are deployed randomly in a large space without boundaries with $K<M$, depicted in Fig. 1 . The $M$ APs communicate simultaneously with the $K$ UEs in the same time/frequency resource. The half duplexing used is time-division duplexing (TDD). It takes advantage from channel reciprocity, which requires a calibration of the hardware chains. As a DL precoding technique, we use the conjugate beamforming. The choice of a such precoder is justified due to (i) its ability to be implemented on a distributed system; and (ii) to avoid the exchange of channel state information (CSI) among APs.

We assume that TDD frame length is equal to the coherence interval length $\tau_{\mathrm{c}}$. We consider $g_{m k}$ the channel coefficient between the $k$-th UE and the $m$-th $\mathrm{AP}$ which is defined as follows:

$$
g_{m k}=\beta_{m k}^{1 / 2} h_{m k},
$$

where $h_{m k}$ represents the small-scale fading, and $\beta_{m k}$ the large-scale fading, which includes pathloss and shadowing. $\beta_{m k}$ coefficients are supposed to be known and $h_{m k}, m=$ $1, \ldots, M, k=1, \ldots, K$, are independent and identically distributed (i.i.d.) $\mathcal{C N}(0,1)$ RVs.

During the UL training phase, via the minimum mean square error (MMSE) estimator, the $m$-th AP estimates the UL channel $\hat{g}_{m k}$ by projecting its received signal onto a UL pilot signal. The latter, sent with full power by the $k$-th UE, is a $\tau_{\mathrm{ul}, \mathrm{p}}$-length sequence and defined by $\sqrt{\tau_{\mathrm{ul}, \mathrm{p}}} \varphi_{k} \in \mathbb{C}^{\tau_{\mathrm{ul}, \mathrm{p}} \times 1}$, $k=1, \ldots, K$ [4]. We suppose that the UL pilot symbols are mutually orthonormal. To this end, one of the sine qua non conditions is that $\tau_{\mathrm{ul}, \mathrm{p}} \geqslant K$, i.e., $\tau_{\mathrm{ul}, \mathrm{p}}=K$ is the smallest number of symbols required to generate $K$ orthogonal pilots. $\hat{g}_{m k} \sim \mathcal{C N}\left(0, \gamma_{m k}\right)$ where

$$
\gamma_{m k} \triangleq \mathbb{E}\left\{\left|\hat{g}_{m k}\right|^{2}\right\}=\frac{\tau_{\mathrm{ul}, \mathrm{p}} \rho_{\mathrm{ul}, \mathrm{p}} \beta_{m k}^{2}}{\tau_{\mathrm{ul}, \mathrm{p}} \rho_{\mathrm{ul}, \mathrm{p}} \beta_{m k}+1},
$$

and $\rho_{u l, p}$ is the normalized transmit signal-to-noise ratio (SNR) related to the pilot symbol.

In the DL data transmission phase, the $m$-th AP uses the $\hat{g}_{m k}$ in order to precode, via the conjugate beamforming, the signal to be transmitted to the $k$-th UE. Hence, the $m$-th AP transmits to all UEs the following signal

$$
x_{\mathrm{dl}, \mathrm{d}, m}^{\mathrm{CF}}=\sqrt{\rho_{\mathrm{dl}, \mathrm{d}}} \sum_{k=1}^{K} \sqrt{\eta_{m k}} \hat{g}_{m k}^{*} q_{k},
$$

where $q_{k}$ is the data symbol intended to the $k$-th UE, which satisfies $\mathbb{E}\left\{\left|q_{k}\right|^{2}\right\}=1$. The symbols $q_{k}$ have zero mean and unit variance, and they are uncorrelated. $\rho_{\mathrm{dl}, \mathrm{d}}$ is the normalized transmit SNR related to the data symbol. Furthermore, $\eta_{m k}$, $m=1, \ldots, M, k=1, \ldots, K$, denote the power control coefficients, which are fixed subject to the average power constraint at each AP as follows

$$
\mathbb{E}\left\{\left|x_{\mathrm{dl}, \mathrm{d}, m}^{\mathrm{CF}}\right|^{2}\right\} \leqslant \rho_{\mathrm{dl}, \mathrm{d}} \Longrightarrow \sum_{k=1}^{K} \eta_{m k} \gamma_{m k} \leqslant 1 \quad \forall m .
$$

The $k$-th UE receives a linear combination of the data signals transmitted by all the APs in (3) as follows:

$$
r_{\mathrm{d}, k}^{\mathrm{CF}}=\sum_{m=1}^{M} g_{m k} x_{\mathrm{dl}, \mathrm{d}, m}^{\mathrm{CF}}+w_{\mathrm{dl}, k}=\sqrt{\rho_{\mathrm{dl}, \mathrm{d}}} \sum_{k^{\prime}=1}^{K} a_{k k^{\prime}} q_{k^{\prime}}+w_{\mathrm{dl}, k},
$$

where $a_{k k^{\prime}} \triangleq \sum_{m=1}^{M} \sqrt{\eta_{m k^{\prime}}} g_{m k} \hat{g}_{m k^{\prime}}^{*}, k^{\prime}=1, \ldots, K$ describes the effective DL channel gain seen by the $k$-th UE for the transmitted symbol to the $k^{\prime}$-th UE, and $w_{\mathrm{dl}, k}$ is additive $\mathcal{C N}(0,1)$ noise at the $k$-th UE. It is primordial that the $k$-th UE have a sufficient knowledge of $a_{k k}$ in order to reliably decode $q_{k}$ as it is revealed in [4].

In the DL training phase, to determine the effective DL channel gain estimate $\hat{a}_{k k}$ via MMSE, each AP precodes the DL pilots, defined by $\boldsymbol{\psi}_{k^{\prime}} \in \mathbb{C}^{\tau_{\mathrm{dllp}, \mathrm{p}}}, k^{\prime}=1, \ldots, K$, via the conjugate beamforming, and beamforms it to all UEs. The DL pilots, assigned randomly from a set of orthogonal sequences of length $\tau_{\mathrm{dl}, \mathrm{p}}<K$, can be either mutually orthonormal or identical. The expression of $\hat{a}_{k k}$ and its proof are shown in details in [7].

\section{PERFORMANCE ANALYSIS}

In the DL, UEs may resort to the statistical CSI (sCSI) or estimate the instantaneous CSI (iCSI) to decode data. Dealing with a fading channel with a non-gaussian noise and a UE having an imperfect iCSI, the UE relies on beamformed DL pilots to estimate the DL channel gain. This latter is considered as a prior information for the capacity-bounding technique as [7. Sec. III-A]. As $\left\{a_{k k^{\prime}}\right\}, k, k^{\prime}=1, \ldots, K$, can 
be approximated as gaussian RVs, a closed-form expression for an approximate achievable DL rate of the transmission from the APs to the $k$-th UE in a CF-mMIMO system with conjugate beamforming, orthogonal UL and non-orthogonal DL pilots, for any finite $M$ and $K$, is given by

$$
R_{k}^{\mathrm{iCSI}, \mathrm{CF}}=\log _{2}\left(1+\frac{W_{\mathrm{DS}_{k}}}{V_{\tilde{a}_{k k}}+\sum_{k^{\prime} \neq k}^{K} W_{\mathrm{IUI}_{k k^{\prime}}}+1}\right),
$$

where $W_{\mathrm{DS}_{k}}=\rho_{\mathrm{dl}, \mathrm{d}}\left(\sum_{m=1}^{M} \sqrt{\eta_{m k}} \gamma_{m k}\right)^{2}+\rho_{\mathrm{dl}, \mathrm{d}} \kappa_{k}$, $W_{\mathrm{IUI}_{k k^{\prime}}}=\rho_{\mathrm{dl}, \mathrm{d}} \zeta_{k k^{\prime}}, V_{\tilde{a}_{k k}}=\rho_{\mathrm{dl}, \mathrm{d}}\left(\zeta_{k k}-\kappa_{k}\right)$ are, respectively, the power of the desired signal, the IUI caused by the $k^{\prime}$-th UE to the $k$-th UE, and the variance of the DL channel estimation error $\tilde{a}_{k k}$, given by $\tilde{a}_{k k}=a_{k k}-\hat{a}_{k k} . \kappa_{k}$, the variance of the DL channel estimate, which includes the effects of the DL pilot contamination, is as follows

$$
\kappa_{k}=\operatorname{Var}\left\{\hat{a}_{k k}\right\}=\frac{\tau_{\mathrm{dl}, \mathrm{p}} \rho_{\mathrm{dl}, \mathrm{p}} \zeta_{k k}^{2}}{1+\tau_{\mathrm{dl}, \mathrm{p}} \rho_{\mathrm{dl}, \mathrm{p}} \sum_{k^{\prime}=1}^{K} \zeta_{k k^{\prime}}\left|\boldsymbol{\psi}_{k}^{H} \boldsymbol{\psi}_{k^{\prime}}\right|^{2}},
$$

and $\zeta_{k k^{\prime}}=\sum_{m=1}^{M} \eta_{m k^{\prime}} \beta_{m k} \gamma_{m k^{\prime}}$. In contrast, when the $k$-th UE relies on sCSI [7, Eq. 35], the achievable DL rate is given by substituting $\kappa_{k}=0$ in 6 .

\section{GRAPH THEORETIC APPROACH FOR DL PILOT ASSIGNMENT}

In essence, we deal with the proposed graph-theoretic approach to allocate DL pilots to all UEs in CF-mMIMO. First, we explain the idea behind the proposed approach and then the graph-theoretic approach is deployed in details.

As it is shown in (6), the DL achievable rate is limited by DL pilot contamination. From (6), we define a symmetric matrix $\Theta=\left[\theta_{k k^{\prime}}\right]_{K \times K}$, whose elements measure the strength of potential IUI between the $k$-th UE and the $k^{\prime}$-th UE, defined as follows

$$
\theta_{k k^{\prime}}=\frac{\mathrm{W}_{\mathrm{IUI}_{k k^{\prime}}}}{\mathrm{W}_{\mathrm{DS}_{k}}}+\frac{\mathrm{W}_{\mathrm{IUI}_{k^{\prime} k}}}{\mathrm{~W}_{\mathrm{DS}_{k^{\prime}}}} .
$$

$\theta$ is expressly the ratio of interference channel strength and the desired signal strength. Larger $\theta_{k k^{\prime}}$ indicates more severe interference between the $k$-th and the $k^{\prime}$-th UEs when the same pilot is allocated to these UEs. It will be wise to define a threshold $\lambda_{\text {th }}$ by which we can decide whether two UEs can reuse the same pilot, i.e., $\theta_{k, k^{\prime}}<\lambda_{\text {th }}$, or not.

\section{A. Construction of Conflict Graph based Pilot Allocation}

In this section, we describe a resource-allocation approach, which exploits the knowledge of dominant DL interference. The proposed method converts the resource allocation problem into a graph coloring problem. Based on $\lambda_{\text {th }}$, we construct a binary adjacency matrix $A=\left[\alpha_{k k^{\prime}}\right]_{K \times K}$, whose entries are defined as follows

$$
\alpha_{k k^{\prime}}= \begin{cases}1, & \text { if } \theta_{k k^{\prime}}>\lambda_{\mathrm{th}} \\ 0, & \text { otherwise }\end{cases}
$$

As $\Theta, A$ is symmetric. Later, the adjacency matrix is used to construct the adjacency list and then the conflict or interference graph is used to describe the interference relationship among all UEs in CF-mMIMO system. Each of the 1's in the adjacency matrix is equivalent to an edge in the conflict graph. Every edge represents a conflict between two vertices (UEs) and distinct vertex colors represent distinct (orthogonal) pilots.

\section{B. The Proposed Algorithm}

Using a graph theoretic approach, the problem of pilot allocation consists in coloring the conflict graph according to these rules: (i) vertices that are joined by an edge are given different colors, i.e., once two UEs are in conflict, we should avoid assigning the same pilot to these UEs; and (ii) the number of colors being used is minimized. Mathematically, the optimization problem of vertex coloring can be formulated as follows:

$$
\min K_{\mathrm{tr}}, \quad \text { s.t. } \quad c_{k} \neq c_{k^{\prime}}, \quad \text { if } \alpha_{k k^{\prime}}=1,
$$

where $K_{\text {tr }}$ is the required number of unique colors (training resources) to color the graph and $c_{k}$ is the color assigned to the $k$-th UE, $k \in\{1,2, \ldots, K\}$. We notice that only $K$ orthogonal pilots are available and there is no restriction for the number of times a pilot (color) can be reused. We conjecture that the problem in 10) is NP-hard leading to prohibitive complexity. As a consequence, we need to turn around heuristic and suboptimal algorithms to solve this problem.

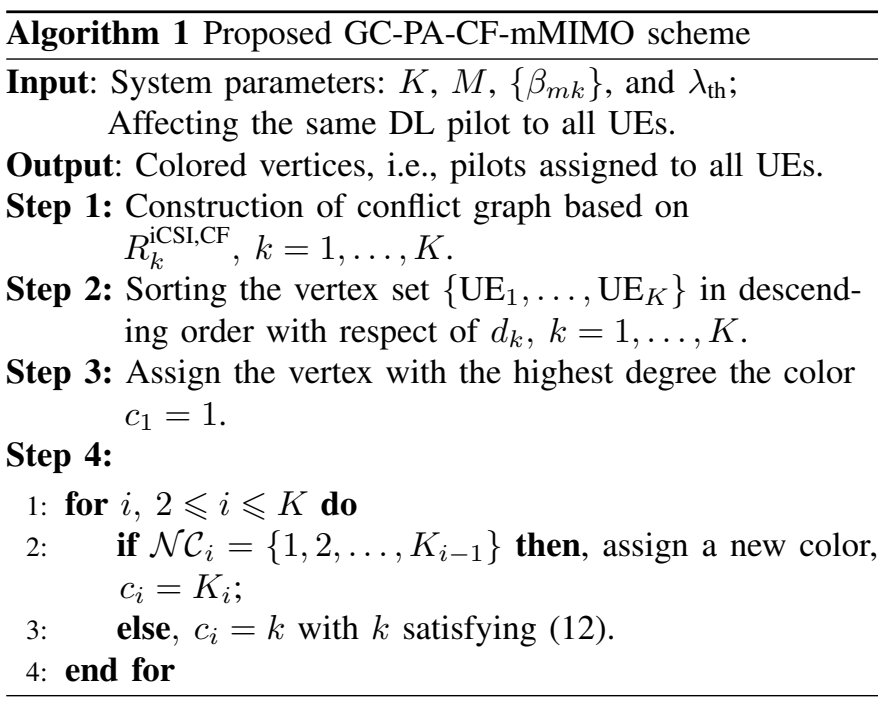

In our work, we use a greedy algorithm to solve the graph coloring problem sequentially shown in Algorithm 1] Its pseudo code can be explained step by step as follows:

As an input, we consider the system parameters $(K, M$, $\left\{\beta_{m k}\right\}$, and $\left.\lambda_{\text {th }}\right)$, and we affect the same DL pilot to all UEs.

1) Step 1: We construct the conflict graph by using $R_{k}^{\mathrm{iCSI}, \mathrm{CF}}$ and the adjacency matrix $A$.

2) Step 2: We denote the $k$-th UE by a vertex $\mathrm{UE}_{k}$. We sort the vertex set $\left\{\mathrm{UE}_{1}, \ldots, \mathrm{UE}_{K}\right\}$ in a descending manner according to the degree of the $k$-th vertex $\mathrm{UE}_{k}$ given by: $d_{k}=\sum_{k^{\prime}=1}^{K} \alpha_{k k^{\prime}}, k=1, \ldots, K$, i.e., the number of edges incident to the vertex $\mathrm{UE}_{k}, k=1, \ldots, K$. In other terms, $d_{k}$ presents the number of UEs in conflict 
with the vertex $\mathrm{UE}_{k}$. The resulting sorted set of step 2 is denoted by $V_{\text {sorted }}$. We note $V_{i}$ an $i$-indexed vertex of the $V_{\text {sorted }}, K_{i}$ is the number of colors used after assignment of colors to the first $i$ vertices and $c_{i}$ is the color used for $V_{i}, \forall 1 \leqslant i \leqslant K$.

3) Step 3: We focus on coloring $V_{1}$. By convention, $c_{1}=1$ and each new color is incremented by one compared to the previous color used as it will be faced in case 1 in step 4. Once $V_{1}$ is colored, we fix $K_{1}=1$.

4) Step 4 (loop for): For each loop iteration $i, \forall i \geq 2$, we color $V_{i}$. To this end, we have to take into consideration the previously colored vertices indexed by the index set $\mathcal{I}_{i}^{\mathrm{c}}=\{1,2, \ldots, i-1\}$. More precisely, the coloring of each vertex $V_{i}$ must respect the following rule to avoid conflict between vertices $V_{i}$ and $V_{k}$,

$$
c_{i} \neq c_{k}, \quad \text { if } \alpha_{i k}=1, \quad \forall k \in \mathcal{I}_{i}^{\mathrm{c}} .
$$

We consider $\mathcal{N C}_{i}$ the set of all colors assigned to vertices in $\mathcal{I}_{i}^{\mathrm{c}}$, which are neighbors with vertex $V_{i}$. To color the latter, two cases arise. The first case is when $\mathcal{N C}_{i}=\left\{1,2, \ldots, K_{i-1}\right\}$, i.e., all previously used colors appear on vertices neighbors with vertex $V_{i}$, then we assign a new color to vertex $V_{i}$ denoted $c_{i}=K_{i}$ where $K_{i}=K_{i-1}+1$. The second case is when the authorized color set to color the vertex $V_{i}$, denoted $\mathcal{A C}_{i}=\left\{1,2, \ldots, K_{i-1}\right\}-\mathcal{N C}_{i}$, is non-empty, then to color vertex $\mathrm{V}_{i}$ we reuse a color $k$, i.e. $c_{i}=k$,

$$
k=\arg \min _{j \in \mathcal{A C}_{i}} n_{j},
$$

where $n_{j}$ is the number of vertices indexed in $\mathcal{I}_{i}^{\mathrm{c}}$ that have been colored by color $j$. In other terms, $k$ is the lowest numbered color that has not been used on any previously colored vertices neighbors with $V_{i}$. Thus, $K_{i}=K_{i-1}<i$. In that case, we prevent excess use of colors (pilots) as we reuse colors.

\section{Threshold Method Selection $\lambda_{t h}$}

The threshold value $\lambda_{\text {th }}$ is applied to determine whether UEs are in conflict or not. If we have $\theta_{k, k^{\prime}}>\lambda_{\text {th }}, \mathrm{UE}_{k}$ and $\mathrm{UE}_{k^{\prime}}$ are in conflict so that they can't reuse the same pilot. If we have $\theta_{k, k^{\prime}} \leq \lambda_{\text {th }}$, $\mathrm{UE}_{k}$ and $\mathrm{UE}_{k^{\prime}}$ are not in conflict so that they can reuse the same pilot. Let's consider the minimum and the maximum of $\theta_{k, k^{\prime}}$, i.e. $\theta_{\min }=\min \left\{\theta_{k, k^{\prime}}\right\}$, $\theta_{\text {max }}=\max \left\{\theta_{k, k^{\prime}}\right\}$. According to the value of $\lambda_{\text {th }}$ chosen, we have different conflict graphs. From (9), it is obvious that $\left.\left.\forall \lambda_{\text {th }} \in\right] \infty, \theta_{\text {min }}\right]$, we have the same conflict graph. Therefore, the lower limit of $\lambda_{\text {th }}$ is $\theta_{\min }$. As well, the upper limit of $\lambda_{\text {th }}$ is $\theta_{\max }$. Thus, $\lambda_{\text {th }} \in\left[\theta_{\min }, \theta_{\max }\right]$.

Let's consider the two following extreme cases. When $\lambda_{\text {th }}$ is fixed to $\theta_{\max }$, there is no conflict between UEs. When we decrease the threshold $\lambda_{\text {th }}$, this leads to a potential conflict between UEs. Thus, the algorithm GC-PA-CF-mMIMO can mitigate the interference with a decreased number of DL pilots (colors). When $\lambda_{\text {th }}$ is fixed to $\theta_{\min }$, the conflict appears among all UEs and the number of pilots (colors) required is $K$ pilots.
In this case, the scheme GC-PA-CF-mMIMO is assimilated to an orthogonal pilot assignment scheme.

To determine a near-optimal threshold $\lambda_{\text {th }}$, we use an iterative grid search (IGS) algorithm as $\lambda_{\text {th }}=$ $f_{\mathrm{IGS}}\left(\left[\theta_{\min }, \theta_{\max }\right], N, T\right)$, where $T$ represents the number of iterations, and $N$ denotes the number of grids per iteration [11]. In short, it consists in selecting the threshold $\lambda_{\text {th }}$ from $N$ grids that maximizes the minimum per-user DL net throughput in every iteration until it reaches $T$ iterations. The description of the algorithm per iteration is as follows. In iteration 1, we consider the $N$ threshold grids for the first iteration by the grid set $\mathcal{G}=\left\{\lambda_{i}^{(1)}: 1 \leqslant i \leqslant N\right\}, \lambda_{0}^{(1)}=\theta_{\min }$, $\lambda_{i+1}^{(1)}=\lambda_{i}^{(1)}+\Delta^{(1)}$ and $\Delta^{(1)}=\frac{\theta_{\max }-\theta_{\min }}{N-1}$. We denote $\lambda_{\max }^{(1)}$ the grid element from the grid set $\mathcal{G}$ that can reach the maximum of the minimum per-user DL net throughput. A sub-interval 1 denoted as $\left[\lambda_{\max }^{(1)}-\Delta^{(1)} / 2, \lambda_{\max }^{(1)}+\Delta^{(1)} / 2\right]$ will be used for the next iteration as a search interval of $\lambda_{\max }^{(2)}$. In iteration $t$, $2 \leqslant t \leqslant T$, once we find the $\lambda_{\max }^{(t)}$, we narrow the search sub-interval $t$ and we get $\left[\lambda_{\max }^{(t)}-\Delta^{(t)} / 2, \lambda_{\max }^{(t)}+\Delta^{(t)} / 2\right]$. This step is performed for $(T-1)$ times. Finally, the near-optimal threshold is as follows: $\lambda_{\text {th }}=\lambda_{\max }^{(T)}$.

\section{NUMERICAL RESULTS AND DISCUSSION}

We evaluate the performance of our proposed solution in terms of per-user downlink net throughput (bit/s) [2] defined by $T_{k}=B \xi^{\mathrm{DL}}\left(1-\frac{\tau_{\mathrm{po}}}{\tau_{\mathrm{c}}}\right) R_{k}$, where $B(\mathrm{~Hz})$ is the spectral bandwidth, $R_{k}$ (bit/s/Hz) is the DL per-user rate, $\xi^{\mathrm{DL}}$ is the fraction of useful samples per coherence interval that are used for DL and $\tau_{\mathrm{po}}=\tau_{\mathrm{ul}, \mathrm{p}}+\tau_{\mathrm{dl}, \mathrm{p}}$ is the pilot overhead.

\section{A. Parameters and setup}

We refer to [4] to model the large-scale fading coefficient $\beta_{m k}$ and the other system parameters. The $M$ APs and $K$ UEs are uniformly and randomly distributed within a square of size $1 \mathrm{~km}^{2}$. To simulate a cell-free topology, i.e., to avoid cell-edge effects, the square simulation area is wrapped around with eight clones of neighbor squares. To have a symmetric TDD frame $\left(\tau_{\mathrm{ul}, \mathrm{d}}=\tau_{\mathrm{dl}, \mathrm{d}}\right)$, we choose $\xi^{\mathrm{DL}}=\frac{1}{2}$. Moreover, to fix the power control coefficients $\eta_{m k}$ at the $m$-th AP, we implement herein a simple equal (uniform) power control policy: $\eta_{m k}=$ $\left(\sum_{k^{\prime}=1}^{K} \gamma_{m k^{\prime}}\right)^{-1} \forall k=1, \cdots, K$, which satisfies the equality condition in (4). This technique, denoted as channel-dependent full power transmission (CD-FPT), is not optimal but it can be performed in a distributive way, and it has a low computational complexity. We suppose that $K \ll \tau_{c}$ and $\tau_{\mathrm{ul}, \mathrm{p}} \tau_{\mathrm{dl}, \mathrm{p}} \geq K$.

\section{B. Results and Discussions}

For UL pilot allocation, we use only orthogonal pilot assignment with $\tau_{\mathrm{ul}, \mathrm{p}}=K$. We deal with an example to show the advantage of our solution in DL pilot assignment comparing to the state-of-the-art. The first prior art is the statistical CSI (sCSI) where no DL training is performed. The second prior art is the instantaneous CSI (iCSI) with orthogonal DL pilots: the DL training is performed for all UEs, and each UE receives an orthogonal DL pilot to estimate the DL channel gain. Hence, for the second prior art, we have 


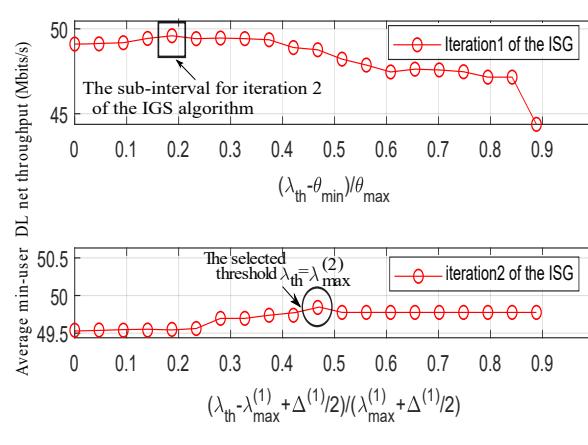

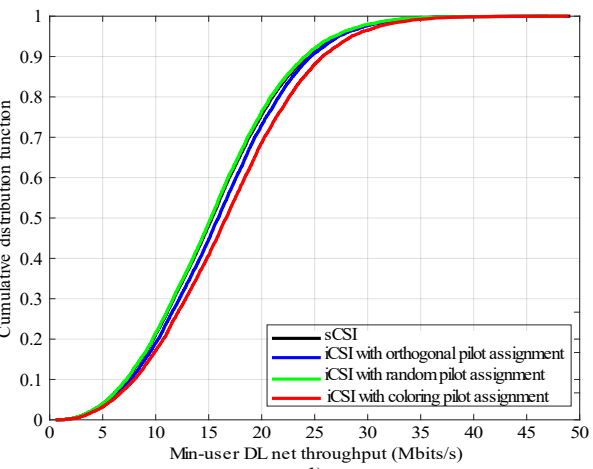

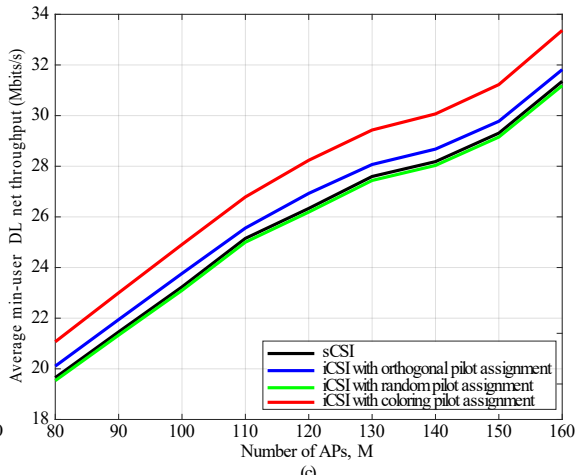

Fig. 2: Simulation results: (a) The IGS algorithm to determine the near-optimal threshold $\lambda_{\text {th }}$ with $M=60, K=10, N=20$ and $T=2$. (b) The CDF of min-user DL net throughput with $M=60, K=10$. (c) The average min-user DL net throughput against the number of APs $M$ with $K=10$.

$\tau_{\mathrm{dl}, \mathrm{p}}^{\text {orth }}=K$. The third prior art is the iCSI with DL random pilot assignment: each UE receives a DL pilot randomly from a set of orthogonal sequences with a length pilot sequence $\tau_{\mathrm{dl}, \mathrm{p}}^{\mathrm{rand}}$, and two UEs may receive the same pilot. For the sake of comparability, we fix $\tau_{\mathrm{dl}, \mathrm{p}}^{\text {rand }}=\tau_{\mathrm{dl}, \mathrm{p}}^{\text {color }}$, where $\tau_{\mathrm{dl}, \mathrm{p}}^{\text {color }}$ is the pilot sequence length used in our proposed scheme.

Fig. 2(a) exemplifies the IGS algorithm to determine the near-optimal threshold $\lambda_{\text {th }}$ with $M=60, K=10, N=20$, and $T=2$. The $\mathrm{X}$-axis represents the normalized threshold interval, and the Y-axis represents the average min-user DL net throughput of the proposed algorithm GC-PA-CF-mMIMO considering the threshold grid set per iteration $\left\{\lambda_{i}^{(t)}: 1 \leqslant i \leqslant\right.$ $N\}$. Once the first iteration is performed, we fix the adequate sub-interval of the IGS algorithm to search $\lambda_{\text {th }}$ with precision within this sub-interval. The near-optimal threshold $\lambda_{\text {th }}=\lambda_{\max }^{(2)}$ can be obtained once the second iteration is performed.

The gain introduced by the proposed scheme in terms of min-user DL net throughput is shown in Fig. 2(b) with $M=60, K=10$. The curves related to the scheme sCSI and the random pilot assignment are almost superimposable. We underline that the DL orthogonal pilot assignment outperforms the DL random pilot assignment by about $0.27 \mathrm{Mbits} / \mathrm{s}$ in concordance with the UL case in [6], and another gain of about $0.83 \mathrm{Mbits} / \mathrm{s}$ can be achieved by the proposed coloring algorithm as it eliminates the potential IUI using the graph conflict and it reduces the number of DL pilots. The proposed scheme outperforms the DL random pilot assignment. The latter strategy doesn't take into consideration the conflict between UEs, so that those in close vicinity may reuse the same DL pilot. This increases DL pilot contamination. The DL orthogonal pilot assignment outperforms the scheme sCSI. As CF-mMIMO is characterized by a channel hardening not sufficiently pronounced, UEs rely on beamformed DL pilots to estimate DL channel with more accuracy [7].

Fig. 2(c) represents the average min-user DL net throughput against $M$ with $K=10$. The performance of prior art and the proposed scheme increase continually when $M$ increases thanks to the diversity gain. Especially, whatever the value of $M$, the proposed scheme outperforms the prior art mentionned before by about $1 \mathrm{Mbits} / \mathrm{s}$.

\section{CONCLUSION}

In conclusion, we proposed a graph-theoretic approach to reduce the DL pilot contamination in CF-mMIMO. First, by choosing an approriate threshold, an interference or conflict graph is dedicated to model the potential interference relationship among all UEs. Then, an algorithm GC-PA-CF-mMIMO is proposed to eliminate the predominant DL interference dealt in the conflict graph. Consequently, using the proposed algorithm GC-PA-CF-mMIMO, the gain in terms of min-user DL net throughput in CF-mMIMO is increased comparing to the conventional DL pilot assignment techniques in CFmMIMO.

\section{REFERENCES}

[1] H. Q. Ngo, A. Ashikhmin, H. Yang, E. G. Larsson, and T. L. Marzetta, "Cell-free Massive MIMO versus small cells," IEEE Trans. Wireless Commun., vol. 16, no. 3, pp. 1834-1850, Mar. 2017.

[2] T. L. Marzetta, E. G. Larsson, H. Yang, and H. Q. Ngo, Fundamentals of Massive MIMO. Cambridge, UK: Cambridge University Press, 2016.

[3] S. Zhou, M. Zhao, X. Xu, J. Wang, and Y. Yao, "Distributed wireless communication system: A new architecture for future public wireless access," IEEE Commun. Mag., vol. 41, no. 3, pp. 108-113, Mar. 2003.

[4] G. Interdonato, P. Frenger and E. G. Larsson, "Utility-based Downlink Pilot Assignment in Cell-Free Massive MIMO," WSA 2018; 22nd International ITG Workshop on Smart Antennas, Mar. 2018, pp. 1-8.

[5] Z. Chen and E. Bjornson, "Channel hardening and favorable propagation in cell-free Massive MIMO with stochastic geometry," IEEE Trans. Commun., vol. 66, no. 11, pp. 5205-5219, Nov. 2018.

[6] M. Bashar, K. Cumanan, A. G. Burr, M. Debbah, and H. Q. Ngo, "On the Uplink Max-Min SINR of Cell-Free Massive MIMO Systems," IEEE Trans. Wireless. Commun., vol. 18, no 4, p. 2021-2036, Apr. 2019.

[7] G. Interdonato, H. Q. Ngo, P. Frenger and E. G. Larsson, "Downlink Training in Cell-Free Massive MIMO: A Blessing in Disguise," IEEE Trans. Wireless Commun, vol. 18, no. 11, pp. 5153-5169, Nov. 2019.

[8] M.Attarifar, A.Abbasfar, A.Lozano, "Random vs structured pilot assignment in cell-free massive MIMO wireless networks." in Proc. IEEE Int'1 Conf. on Commun. Workshops (ICCW'18), May. 2018, pp. 1-6.

[9] E. Björnson and L. Sanguinetti, "A new look at cell-free Massive MIMO: Making it practical with dynamic cooperation,"in Proc. IEEE 30th Annu. Int. Symp. Pers., Indoor, Mobile Radio Commun. (PIMRC), Istanbul, Turkey, Sep. 2019, pp. 1-6.

[10] N. Rupasinghe, Y. Kakishima, H. Papadopoulos, and I. Guvenc, "A graph theoretic approach for training overhead reduction in FDD massive MIMO systems," in Proc. IEEE Int. Commun. Conf. (ICC), May 2018, pp. 1-7.

[11] X. Zhu, L. Dai, and Z. Wang, "Graph coloring based pilot allocation to mitigate pilot contamination for multi-cell massive MIMO systems," IEEE Commun. Letters, vol. 9, no. 10, pp. 1842-1845, Oct. 2015. 